\title{
Spin Localization of a Fermi Polaron in a Quasirandom Optical Lattice
}

Received: 9 November 2016 / Accepted: 23 December 2016 / Published online: 20 January 2017

(C) The Author(s) 2017. This article is published with open access at Springerlink.com

\begin{abstract}
Recently, the topics of many-body localization (MBL) and one-dimensional strongly interacting fewbody systems have received a lot of interest. These two topics have been largely developed separately. However, the generality of the latter as far as external potentials are concerned-including random and quasirandom potentials - and their shared spatial dimensionality, makes it an interesting way of dealing with MBL in the strongly interacting regime. Utilising tools developed for few-body systems we look to gain insight into the localization properties of the spin in a Fermi gas with strong interactions. We observe a delocalized-localized transition over a range of fillings of a quasirandom lattice. We find this transition to be of a different nature for low and high fillings, due to the diluteness of the system for low fillings.
\end{abstract}

\section{Introduction}

Strongly interacting one-dimensional quantum systems have attracted major attention in recent years [15]. When confined to one dimension the fermionic system exhibits a spin-charge separation, and for very strong interactions the charge degrees of freedom are frozen, making it possible to write an effective spin chain Hamiltonian for the system [2-4]. Methods have been developed to solve numerically for the exchange coefficients of this spin chain for any given confining potential $[3,6]$.

The presence of disorder in an interacting system can result in the violation of the eigenstate thermalization hypothesis, due to many-body localization (MBL) [7,8]. The localization of single-particle states in the presence of disorder in quantum systems was originally considered by Anderson [9]. Over the intervening decades, Anderson localization has been observed in many systems, including in electron gases [10], photonic lattices [11], and cold atoms [12]. For a MBL phase in the tight-binding approximation all eigenstates of the system are Anderson localized [7]. Theoretical work on MBL has been focused on the nature of the delocalizationlocalization phase transition as disorder is increased [13-16]. Quantum spin chains have been fruitful models for looking at this transition. In most cases the disorder is introduced in the external magnetic field or coupling coefficients of the spin chain. In this work we still consider a quantum spin chain, but one that is induced by the strong interactions present between fermions. We introduce disorder in the system via a quasirandom optical lattice potential.

In recent years, the field of ultracold atomic gases in one dimension has received a lot of interest [17-19]. Such systems have been considered for strongly interacting fermions [20] and bosons [21-23]. In this field,

This article belongs to the Topical Collection "The 23rd European Conference on Few-Body Problems in Physics".

C. W. Duncan $(\varangle) \cdot$ P. Öhberg · M. Valiente

SUPA, Institute of Photonics and Quantum Sciences, Heriot-Watt University, Edinburgh EH14 4AS, UK

E-mail: cd130@hw.ac.uk

N. J. S. Loft · N. T. Zinner

Department of Physics and Astronomy, Aarhus University, 8000 Aarhus C, Denmark 
the MBL phase transition has been observed with interacting fermions in a one dimensional quasirandom optical lattice [24]. Recently, an experimental realization of only a few strongly interacting fermions in a one dimensional trap has been realised [25].

\section{Model}

We consider $N$ strongly interacting repulsive spin-1/2 fermions in one dimension (see Fig. 1d). This system is described by the Hamiltonian

$$
H=\sum_{i=1}^{N}\left(\frac{p_{i}^{2}}{2 m}+V\left(x_{i}\right)\right)+g \sum_{i<j}^{N} \delta\left(x_{i}-x_{j}\right)
$$

where $g$ is the contact interaction strength, and $V\left(x_{i}\right)$ is the single-particle external potential. We consider the limit of strong repulsive interactions, $g \rightarrow+\infty$, for which the system can be mapped onto an effective spin chain model. We will elaborate on this below. Throughout this paper we set $\hbar=m=1$, and express length in units of the length of the system $L$.

Quasirandom, or quasiperiodic potentials have been shown to exhibit a localization transition for single particle [26,27], and many-body systems [28], as is the case for truly random disorder. Such potentials can be implemented in ultracold atom set-ups [29], and have been used to observe both Anderson localization [30], and MBL [24]. We consider a quasirandom potential with open boundary conditions, with a main lattice of strength $V_{1}$ and a disorder term of strength $V_{2}$. The potential $V(x)$, appearing in Eq. (1) is given by

$$
V(x)=V_{1} \cos \left(\frac{\tau_{1} x}{d}\right)+V_{2} \cos \left(\frac{\tau_{2} x}{d}+\phi\right),
$$

where $d$ is the lattice spacing, defined as $d \equiv L / L_{s}$ with $L_{s}$ giving the number of wells—or 'sites'—in the lattice. Throughout this work we set $\tau_{1}=2 \pi$ and $\tau_{2}=1$, satisfying the need for $\tau_{1} / \tau_{2}$ to be incommensurate for the above potential to be quasirandom. We fix the number of lattice wells $L_{s}=12$, and sweep across the lattice filling $\left(v \equiv N / L_{s}\right)$ by varying the number of particles $N=6,7,8, \ldots, 24$. We will quantify the disorder strength by the ratio $W=V_{2} / V_{1}$, and consider the disorder range of $0 \leq W \leq 1$, with examples of the potential shown in Fig. 1a-c. The main lattice strength $V_{1}=5$ is chosen to ensure that the lattice is strong enough to be felt by all particles, without the particles being localized into single sites.

In the case of strong repulsion, $g \rightarrow \infty$, the system of trapped cold atoms can be described by an effective spin chain model $[2-4,6]$. Specifically, to linear order in $1 / g \ll 1$ the (ground state manifold) spectrum is given by

$$
E_{n}=E_{0}-\frac{K_{n}}{g}
$$

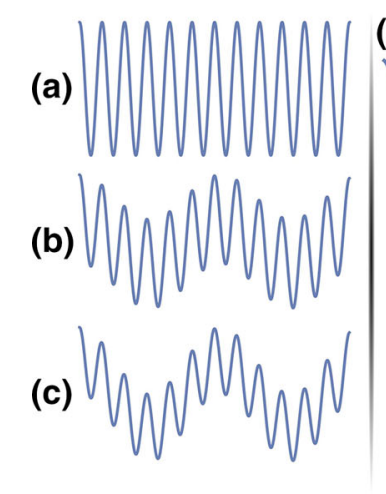

(d)

(d) (e)

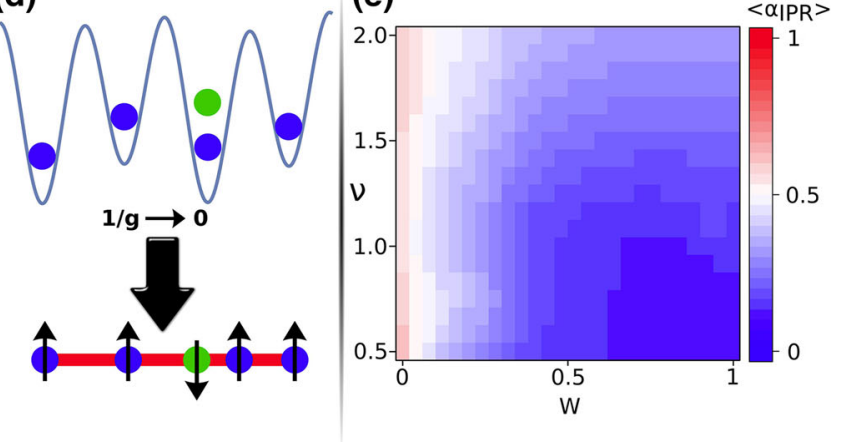

Fig. 1 a-c The quasirandom potential, Eq. (2), for $W=0,0.5$ and 1 respectively. $\mathbf{d}$ Illustration of the mapping to an effective spin chain model for strong interactions. e Average inverse participation ratio for a filling $v=N / L_{s}$ of the $N$ single particle states for disorder strength $W$ 
with $E_{0}$ being the degenerate many-body ground state energy at infinitely strong repulsion $1 / g=0$. Here $K_{n}$ are the eigenstates of the spin chain Hamiltonian

$$
K=-\frac{1}{2} \sum_{j=1}^{N-1} J_{j}\left(\sigma_{j} \cdot \sigma_{j+1}-1\right),
$$

with $\sigma_{j}$ being the Pauli matrices acting on the $j$ th site (or atom) of the spin chain, and $J_{j}$ is the coefficient connecting the $j$ and $j+1$ sites. The spin chain coefficients $J_{j}$ are solely dependent on the single-particle wavefunctions, which are found by solving the stationary Schrödinger equation with the single-particle Hamiltonian $H_{0}=p^{2} / 2 m+V(x)$. Thus different realizations of the quasirandom potential $V(x)$ will translate into variations in the spin chain coefficients for the effective spin chain (4). We use the open source program CONAN [6], which has been developed to take arbitrary potentials and numerically calculate the $N-1$ coefficients $J_{j}$ between the spin chain sites for up to $N \sim 30$ particles. Notice that in this approach, we study the spin chain model resulting from every single realization of the quasirandom potential. The above spin chain model is a pertubative description that is exact to linear order - therefore variational-in $1 / \mathrm{g}$ of the ground state manifold, with two assumptions: Firstly strong repulsion, and secondly zero-temperature. Recently, the formation of an effective spin chain in such limits has been confirmed with agreement between numerics and an experimental system using only a few cold atoms in a one-dimensional harmonic trap [25].

For the numerical investigations we compute the spin chain coefficients $J_{j}$, using CONAN [6], arising from the lattice potential in Eq. (2) for $W$ between 0 and 1, and over a range of particle numbers $N=6,7,8, \ldots, 24$, corresponding to fillings $v \equiv N / L_{s}=1 / 2,0.583,0.667, \ldots, 2$. For each $W$ and $N$ we average over 19 realizations of the phase $\phi$. Using the calculated spin chain coefficients, we solve the stationary Schrödinger equation for the spin chain Hamiltonian, Eq. (4), numerically. For the polaron we will denote the wavefunction as

$$
|\Psi\rangle=\sum_{j=1}^{N} C_{j}\left|\uparrow \ldots \uparrow(\downarrow)_{j} \uparrow \ldots \uparrow\right\rangle,
$$

where $C_{j}$ is the coefficient for the polaron in the $j$ th spin chain site. To gain further insight, we will also consider the case of two polarons, which we expect to have similar general behaviour to the single polaron in this system. For two polarons we write the wavefunction as

$$
|\Psi\rangle=\sum_{i<j}^{N} C_{(i, j)}\left|\uparrow \ldots \uparrow(\downarrow)_{i} \uparrow \ldots \uparrow(\downarrow)_{j} \uparrow \ldots \uparrow\right\rangle .
$$

\section{Measures of Localization}

The onset of Anderson localization in the system can be observed by considering the inverse participation ratio (IPR) [5], given by

$$
\alpha_{\mathrm{IPR}}=\frac{1}{m \sum_{v}\left|C_{v}\right|^{4}},
$$

with $C_{v}$ being the coefficients of either the polaron $v=j$ or two polaron $v=(i, j)$ states, and $m$ denoting the size of the Hilbert space of the wavefunction. For a fully delocalized state $\alpha_{\mathrm{IPR}} \sim 1$. For a fully localized state we have a convergence of $\alpha_{\text {IPR }}$ towards zero. We will consider the IPR of the ground, and the highest energy states. In addition we calculate the average IPR across all other states (denoted by $\left\langle\alpha_{\text {IPR }}\right\rangle$ ). The average IPR gives an indication of the overall localization of the system. However, this is not an exact measure of the localization of all states, e.g. there could be a few heavily localized states with the rest delocalized.

A standard measure of the MBL transition is the properties of the energy level statistics, which can be investigated via the ratio of adjacent energy level gaps $[5,15,31,32]$

$$
r_{n}=\frac{\min \left(\delta_{n}, \delta_{n-1}\right)}{\max \left(\delta_{n}, \delta_{n-1}\right)},
$$

where $\delta_{n}=E_{n}-E_{n-1}$ is the gap in the spectrum between the $E_{n}$ and $E_{n-1}$ eigenvalues, with min and max taking the minimum and maximum value of the two adjacent gaps in the spectum $\left(\delta_{n}, \delta_{n-1}\right)$, ensuring 
$0 \leq r_{n} \leq 1$. We will take the average of the gap ratio $\left\langle r_{n}\right\rangle$ over all $\delta_{n}$, with the exclusion of the ground and highest energy states as will be discussed in Sect. 4. In the delocalized phase we expect the energy level statistics to satisfy a Wigner-Dyson disitribution (WD) [15,31], with the average ratio $\left\langle r_{n}\right\rangle_{\mathrm{WD}} \simeq 0.536$ [5]. Meanwhile, the MBL phase has statistics that satisfy the Poisson distribution (PD) [15,31], with an average ratio of $\left\langle r_{n}\right\rangle_{\mathrm{PD}} \simeq 0.386$ [5].

\section{Localization of the Spin}

First we confirm the localization in the charge degree of freedom. In Fig. 1e, we consider the average IPR over all single particle states for the quasirandom lattice, Eq. (2). We find for disorder above $W \sim 0.5$ the general localization of the single particle states across the whole range of $v$. The critical disorder for the delocalized to localized transition of the single particle states is weakly dependent on the filling of the lattice. This result gives a good indication that the particles are "feeling" the lattice potential.

For the spin of a single polaron we consider $\alpha_{\mathrm{IPR}}$ of the ground, and highest energy states, then $\left\langle\alpha_{\mathrm{IPR}}\right\rangle$ over all other states, with the results shown in Fig. 2. In addition, we consider two polarons in the system, with the same observables as for the single polaron, but in addition we calculate the average energy gap ratio $\left\langle r_{n}\right\rangle$. We do not consider the gap ratio for the single polaron due to the small number of states, $N$, in the system, resulting in large variances in $\left\langle r_{n}\right\rangle$ over the realizations of the disorder. Naturally, for two polarons there is a larger number of states, $N(N-1) / 2$, resulting in smaller variance over the disorder realizations.

The groundstate of the spin is found to localize at small disorder in Figs. 2a and 3a, with strong localization for $W>0.1$, for most $v$. With the exception of around unit filling, where we have a spin in each lattice site, resulting in an elongated transition to the localized state. The highest energy state is delocalized across the system over all disorder, Figs. $2 \mathrm{~b}$ and $3 \mathrm{~b}$. Therefore, for our system we can never have a true MBL phase, in the sense that all states will not localize. However, with the inherently delocalized highest energy state excluded, we observe a delocalized-localized transition over a range of $v$.

With the average IPR over all states in the system except the ground and highest states, $\left\langle\alpha_{\mathrm{IPR}}\right\rangle$, we can gain an insight into the general localization properties of the system, see Figs. 2c and 3c. We observe a defined transition from a majority of states being delocalized to heavily localized over a range of fillings from $1 \leq v \leq 2$. For small fillings, $v<1$, we observe a trend towards localization with large disorder. The relatively weak localization of states at these fillings is due to the diluteness of the system. Each fermion (or groups
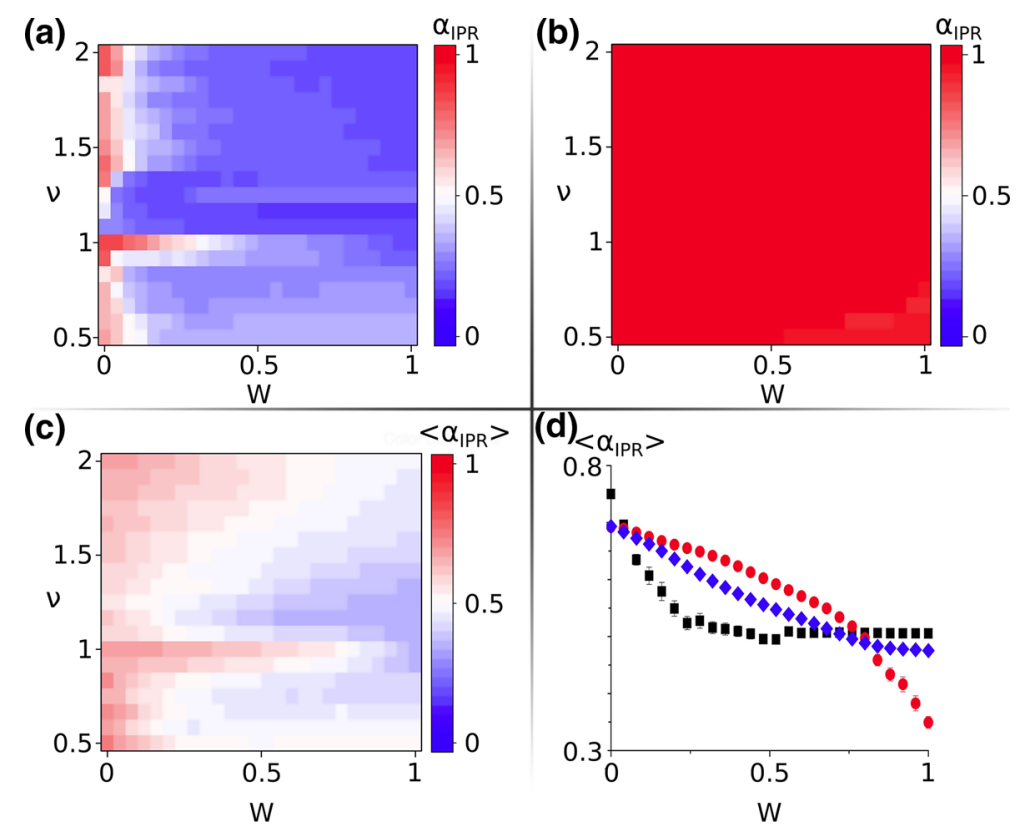

Fig. $2 \alpha_{\text {IPR }}$ for the single polaron spin chain. a Groundstate IPR. b Highest energy state IPR. c The average IPR over all states except the ground and highest, $\left\langle\alpha_{\text {IPR }}\right\rangle$. d Cut-outs of $\mathbf{c}$ for $v=0.5$ squares (black), $v=1$ circles (red), and $v=2$ diamonds (blue) 

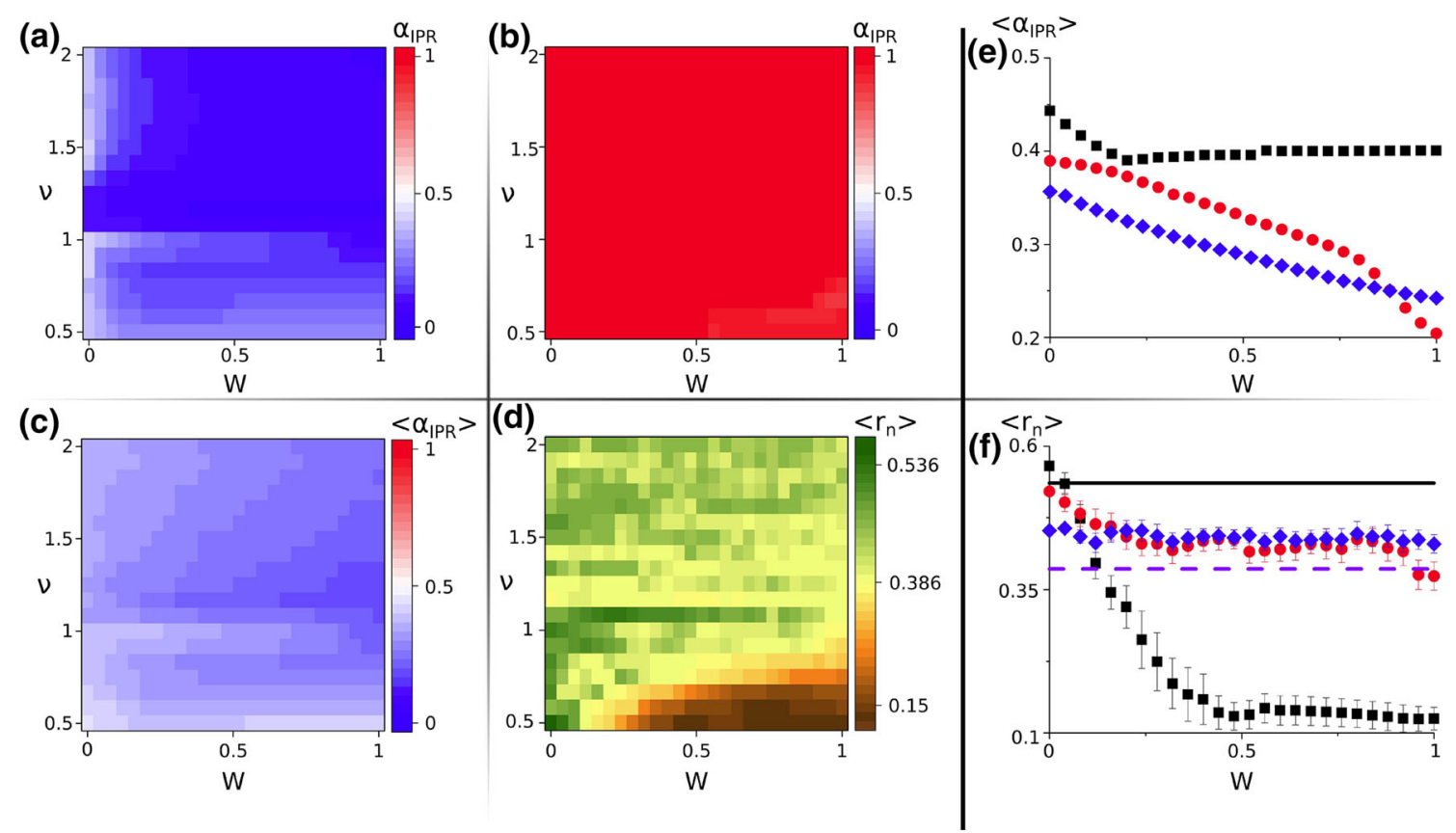

Fig. $3 \alpha_{\text {IPR }}$ for the two-polaron spin chain. a Groundstate IPR, averaged over 9 disorder phase, $\phi$, realizations. b Same as (a) but for the highest energy state. $\mathbf{c}$ The average IPR over all states except the ground and highest, $\left\langle\alpha_{\mathrm{IPR}}\right\rangle$. $\mathbf{d}$ The energy gap ratio, $\left\langle r_{n}\right\rangle$. $\mathbf{e}$ and $\mathbf{f}$ cut-outs of $\mathbf{c}$ and $\mathbf{d}$ respectively for $v=0.5$ squares (black), $v=1$ circles (red), and $v=2$ diamonds (blue). On plot $\mathbf{f}$ black solid line and purple dashed line denote the expected values for an extended and localized phase respectively

of fermions) can be well seperated from its neighbours, resulting in weak coupling coefficients, effectively resulting in the separation of the spin chain into sections. Hence we observe some localization of the state, but not due to disorder in the spin chain. The regimes discussed are well shown by the cut outs of Figs. $2 \mathrm{~d}$ and $3 \mathrm{e}$.

However, the IPR is a poor measure of the localization of all states, and a standard measure for this (the MBL phase) is the average energy gap ratio in the spectrum, $\left\langle r_{n}\right\rangle$. We calculated $\left\langle r_{n}\right\rangle$ for two polarons in the system, Fig. 3d, with a cut out at select fillings in Fig. 3f. For $v \sim 1$, we see a transition from an extended $\left(\left\langle r_{n}\right\rangle_{\mathrm{WD}} \simeq 0.536\right)$ to a localized phase $\left(\left\langle r_{n}\right\rangle_{\mathrm{PD}} \simeq 0.386\right)$. At $v=1.0833=1+1 / 12$, where we are at one particle over unit filling, we observe the states to have Poisson statistics independent of disorder, shown by the yellow region above unit filling in Fig. 3d. This is due to the spin chain coefficients having a form that is 'well-like' at this filling without the prescence of disorder [33]. Thus the statistics of the eigenvalue gaps are that of the Poisson distribution, as has been shown for interacting trapped bosons in harmonic potentials [34]. For higher filling, we see a transition from a delocalized to a localized phase with increasing disorder. However as we approach $v=2,\left\langle r_{n}\right\rangle$ is consistently at an intermediate value, Fig. 3f, which is consistent with a mixed phase of localized and delocalized states.

With $v<1$, we observe a different regime of the system. $\left\langle r_{n}\right\rangle$ converges to a value well bellow 0.386 , as seen in Fig. 3d, f, with a weak localization across all states as seen in Figs. $2 \mathrm{c}$ and $3 \mathrm{c}$. The convergence value of $\left\langle r_{n}\right\rangle$ is not consistent with any spectrum we know of. For $v<1$ the states are localized because of the break up of the spin chain due to the diluteness of the system, and not through disorder. The gap ratio further reflects the different nature of the localization transition of the states for low filling.

\section{Conclusions}

Using recent advances in describing strongly interacting confined particles in one dimension, we have investigated the localization properties of the spin degree of freedom. It is well known that the charge degree of freedom is localized in this system in the presence of strong interactions. For the spin we observe the localization of the majority of states upon sufficient disorder for $v>1$. For small fillings, $v<1$, we observe a weak localization regime due to the system being dilute. The system considered can never be completely localized, due to the presence of a fully delocalized highest energy state. This state is an inherent property of the system. 
However with the exclusion of this delocalized state, we observe a delocalized to localized transition for both the polaron and two polaron systems. This transition is seen for fillings above unity by the convergence of the level statistics to the Poisson distribution expected in the MBL phase. For low fillings and above a certain disorder strength we see the emergence of a regime with different statistics, due to the diluteness of the system.

Acknowledgements C.W.D. acknowledges support from EPSRC CM-CDT Grant No. EP/L015110/1. P.Ö. and M.V. acknowledge support from EPSRC EP/M024636/1. N. J. S. L. and N. T. Z. acknowledge support by the Danish Council for Independent Research DFF Natural Sciences and the DFF Sapere Aude program.

Open Access This article is distributed under the terms of the Creative Commons Attribution 4.0 International License (http:// creativecommons.org/licenses/by/4.0/), which permits unrestricted use, distribution, and reproduction in any medium, provided you give appropriate credit to the original author(s) and the source, provide a link to the Creative Commons license, and indicate if changes were made.

\section{References}

1. M.L. Wall, L.D. Carr, Strongly interacting fermions in an optical lattice. Phys. Rev. A 87, 033601 (2013)

2. A.G. Volosniev, D.V. Fedorov, A.S. Jensen, M. Valiente, N.T. Zinner, Strongly interacting confined quantum systems in one dimension. Nat. Commun. 5, 5300 (2014)

3. F. Deuretzbacher, D. Becker, J. Bjerlin, S.M. Reimann, L. Santos, Quantum magnetism without lattices in strongly interacting one-dimensional spinor gases. Phys. Rev. A 90, 013611 (2014)

4. A.G. Volosniev, D. Petrosyan, M. Valiente, D.V. Fedorov, A.S. Jensen, N.T. Zinner, Engineering the dynamics of effective spin-chain models for strongly interacting atomic gases. Phys. Rev. A 91, 023620 (2015)

5. Hu Hui, An-Bang Wang, Su Yi, Xia-Ji Liu, Fermi polaron in a one-dimensional quasiperiodic optical lattice: the simplest many-body localization challenge. Phys. Rev. A 93, 053601 (2016)

6. N.J.S. Loft, L.B. Kristensen, A.E. Thomsen, A.G. Volosniev, N.T. Zinner, CONAN-the cruncher of local exchange coefficients for strongly interacting confined systems in one dimension. Comput. Phys. Commun. 209, 171-182 (2016)

7. R. Nandkishore, D.A. Huse, Many-body localization and thermalization in quantum statistical mechanics. Annu. Rev. Condens. Matter Phys. 6(1), 15-38 (2015)

8. E. Altman, R. Vosk, Universal dynamics and renormalization in many-body-localized systems. Annu. Rev. Condens. Matter Phys. 6(1), 383-409 (2015)

9. P.W. Anderson, Absence of diffusion in certain random lattices. Phys. Rev. 109, 1492-1505 (1958)

10. Melvin Cutler, N.F. Mott, Observation of Anderson localization in an electron gas. Phys. Rev. 181, 1336-1340 (1969)

11. M. Segev, Y. Silberberg, D.N. Christodoulides, Anderson localization of light. Nat. Photon. 7, 197-204 (2013)

12. J. Billy, V. Josse, Z. Zuo, A. Bernard, B. Hambrecht, P. Lugan, D. Clement, L. Sanchez-Palencia, P. Bouyer, A. Aspect, Direct observation of Anderson localization of matter waves in a controlled disorder. Nature 453, 891-894 (2008)

13. Arijeet Pal, David A. Huse, Many-body localization phase transition. Phys. Rev. B 82, 174411 (2010)

14. Jonas A. Kjäll, Jens H. Bardarson, Frank Pollmann, Many-body localization in a disordered quantum Ising chain. Phys. Rev. Lett. 113, 107204 (2014)

15. Maksym Serbyn, Z. Papić, D.A. Abanin, Criterion for many-body localization-delocalization phase transition. Phys. Rev. X 5, $041047(2015)$

16. John Z. Imbrie, Diagonalization and many-body localization for a disordered quantum spin chain. Phys. Rev. Lett. 117, 027201 (2016)

17. Immanuel Bloch, Ultracold quantum gases in optical lattices. Nat. Phys. 1, 23-30 (2005)

18. Immanuel Bloch, Jean Dalibard, Wilhelm Zwerger, Many-body physics with ultracold gases. Rev. Mod. Phys. 80, 885-964 (2008)

19. Xi-Wen Guan, Murray T. Batchelor, Chaohong Lee, Fermi gases in one dimension: From Bethe ansatz to experiments. Rev. Mod. Phys. 85, 1633-1691 (2013)

20. Guido Pagano, Marco Mancini, Giacomo Cappellini, Pietro Lombardi, Florian Schafer, Hu Hui, Xia-Ji Liu, Jacopo Catani, Carlo Sias, Massimo Inguscio, Leonardo Fallani, A one-dimensional liquid of fermions with tunable spin. Nat. Phys. 10, 198-201 (2014)

21. Toshiya Kinoshita, Trevor Wenger, David S. Weiss, Observation of a one-dimensional Tonks-Girardeau gas. Science 305(5687), 1125-1128 (2004)

22. Belen Paredes, Artur Widera, Valentin Murg, Olaf Mandel, Simon Folling, Ignacio Cirac, Gora V. Shlyapnikov, Theodor W. Hänsch, Immanuel Bloch, Tonks-Girardeau gas of ultracold atoms in an optical lattice. Nature 429, $277-281$ (2004)

23. A. Lazarides, O. Tieleman, C. Morais Smith, Strongly interacting bosons in a one-dimensional optical lattice at incommensurate densities. Phys. Rev. A 84, 023620 (2011)

24. Michael Schreiber, Sean S. Hodgman, Pranjal Bordia, Henrik P. Lüschen, Mark H. Fischer, Ronen Vosk, Ehud Altman, Ulrich Schneider, Immanuel Bloch, Observation of many-body localization of interacting fermions in a quasirandom optical lattice. Science 349(6250), 842-845 (2015)

25. S. Murmann, F. Deuretzbacher, G. Zürn, J. Bjerlin, S.M. Reimann, L. Santos, T. Lompe, S. Jochim, Antiferromagnetic Heisenberg spin chain of a few cold atoms in a one-dimensional trap. Phys. Rev. Lett. 115, 215301 (2015)

26. D.R. Grempel, S. Fishman, R.E. Prange, Localization in an incommensurate potential: An exactly solvable model. Phys. Rev. Lett. 49, 833-836 (1982)

27. S. Ganeshan, J.H. Pixley, S. Das Sarma, Nearest neighbor tight binding models with an exact mobility edge in one dimension. Phys. Rev. Lett. 114, 146601 (2015) 
28. S. Iyer, V. Oganesyan, G. Refael, D.A. Huse, Many-body localization in a quasiperiodic system. Phys. Rev. B 87, 134202 (2013)

29. L. Fallani, C. Fort, M. Inguscio, Bose-Einstein condensates in disordered potentials, in Advances in Atomic, Molecular, and Optical Physics, ed. by E. Arimondo, P.R. Berman, C.C. Lin. vol. 56 (Academic Press, 2008), pp. 119-160

30. G. Roati, C. D'Errico, L. Fallani, M. Fattori, C. Fort, M. Zaccanti, G. Modugno, M. Modugno, M. Inguscio, Anderson localization of a non-interacting Bose-Einstein condensate. Nature 453, 895-898 (2008)

31. Vadim Oganesyan, David A. Huse, Localization of interacting fermions at high temperature. Phys. Rev. B 75, 155111 (2007)

32. Rubem Mondaini, Marcos Rigol, Many-body localization and thermalization in disordered hubbard chains. Phys. Rev. A 92 , $041601(2015)$

33. C Duncan, F Bellotti, P Öhberg, N T Zinner, M Valiente, Spin traps for Fermi polarons in one-dimensional lattices (in preperation)

34. Barnali Chakrabarti, Anindya Biswas, V.K.B. Kota, Kamalika Roy, Sudip Kumar Haldar, Energy-level statistics of interacting trapped bosons. Phys. Rev. A 86, 013637 (2012) 